\title{
FOCUSED ION BEAM MICROMACHINING OF GaN PHOTONIC DEVICES
}

\author{
Irving Chyr and A. J. Steckl \\ Nanoelectronics Laboratory, University of Cincinnati \\ Cincinnati, Ohio 45221-0030 USA, a.steckl@uc.edu \\ Cite this article as: MRS Internet J. Nitride Semicond. Res. 4S1, G10.7 (1999)
}

\begin{abstract}
$\mathrm{Ga}^{+}$and $\mathrm{Au}^{+}$focused ion beams (FIB) are used to micromachine GaN films. The GaN micromachining has been studied at energies from $30-90 \mathrm{keV}$, incident angle from $0-30^{\circ}$, and number of repetitive scans from 10 to 50 scans. Trenches milled in $\mathrm{GaN}$ have vertical and smooth side-walls and very smooth bottoms. The micromachining rate was found to be fairly independent of ion dose, ranging from 0.4 to $0.6 \mu \mathrm{m}^{3} / \mathrm{nC}$ for $\mathrm{Ga}^{+}$and 1 to $2 \mu \mathrm{m}^{3} / \mathrm{nC}$ for $\mathrm{Au}^{+}$. This translates into an effective yield of of 6-7 atoms/ion for $\mathrm{Ga}^{+}$and 21-26 atoms/ion for $\mathrm{Au}^{+}$. This represents the highest direct FIB removal yield reported to date. We have also investigated the micromachining of $\mathrm{GaN}$ substrate material: c-face sapphire. Using FIB $\mathrm{Ga}^{+}$, sapphire has an effective yield of $\sim 2-2.5$ atoms/ion, or approximately $1 / 3$ of the GaN sputtering yield. For the materials investigated, we found the sputtering yield to be inversely proportional to the strength of the material chemical bond. We also describe the application of the FIB $\mu$ machining technique to the fabrication of small period Distributed Bragg Reflector (DBR) mirrors for a short cavity GaN laser structure.
\end{abstract}

\section{INTRODUCTION}

GaN and its alloys are of great interest for visible and UV light emitting devices because of their applications for displays, scanners, printers, optical disks, etc. Recently, commercialized laser diodes ${ }^{1}$ have been announced using GaN-based structure grown on sapphire and $\mathrm{SiC}$ substrate. Most of the research and development to date is focused on GaN grown on sapphire substrates because of good crystal quality. However, there still remain some fabrication issues concerning sample preparation and processing. In particular, suitable cavity and high reflectivity mirror facets are hard to obtain by conventional processing procedures due to the large misalignment ${ }^{2}$ between sapphire and GaN-based materials. Currently, facets are formed by either the cleaving method ${ }^{3}$ (which cannot provide lower roughness on the sidewalls), or by dry plasma etching 4 (which generates high ion-induced damage and smooth etched sidewalls). Therefore, it is important to find a simple and efficient processing technique in order to fabricate low mirror loss and mode selection of laser diodes.

Conventional edge-emitting semiconductor lasers fabricated by cleaving result in the low mirror reflectivity in the range of $\sim 0.3-0.4$. Even though there are distributed feedback (DFB) or distributed Bragg reflector (DBR) ${ }^{5,6}$ in the ends of the laser cavity, the overall mirror reflectivity still remains low. Recently several research groups ${ }^{7,8,9}$ reported an alternative method to increase the mirror reflectivity of semiconductor lasers by introducing a short (but deep) stack of DBR grating consisting of multilayers of semiconductor materials and air. This is attractive since 
the large refractive index difference between semiconductor materials and air provides a very high reflectivity and reduces the length of cavity at the same time.

The Focused Ion Beam (FIB) technique has been applied to many aspects of microelectronic device fabrication. In particular, FIB is successfully used in the areas of micromachining ${ }^{10}$ and ion-induced mixing ${ }^{11}$, with applications to photonic components such as waveguides, mirrors, gratings and laser cavity in the GaAs/AlAs and InP materials systems. Recently, there are several groups ${ }^{12,13}$ who have applied FIB micromachining to produce flat and smooth mirror facets for $\mathrm{GaN}$ laser diodes. Nonetheless, no quantitative analysis of $\mathrm{GaN}$ micromachining has been reported to date. In this paper, we present the results on FIB micromachining of $\mathrm{GaN}$ and related substrates under various experimental parameters of energy, incident angle and scan strategy. We also apply FIB micromachining for the fabrication of GaN laser with short-period cavity with semiconductor/air DBR mirrors.

\section{EXPERIMENT}

Liquid metal ion source (LMIS) of $\mathrm{Ga}^{+}$and $\mathrm{Au}^{+}$were used with the MBI (MicroBeam Inc.) NanoFab 150 system of FIB. This system has two sets of electrostatic lenses and $\mathrm{E} \times \mathrm{B}$ mass separation. The total emission current was typically set at $3 \mu \mathrm{A}$ for $\mathrm{Ga}^{+}$and $15 \mu \mathrm{A}$ for $\mathrm{Au}^{+}$. The target current of $\mathrm{Ga}^{+}$was $\sim 170 \mathrm{pA}$ for measuring milling rate, $\sim 220 \mathrm{pA}$ for fabricating the waveguides and $\sim 22 \mathrm{pA}$ for grating fabrication. The $\mathrm{Au}^{+}$target current was $\sim 185 \mathrm{pA}$ for measuring milling rate. The total dose used in the experiments for the milling rate analysis reported here was kept constant at $1 \times 10^{18} \mathrm{~cm}^{-2}$. We used $2.5 \times 10^{18} \mathrm{~cm}^{-2}$ for waveguide and gratings. Multiple number of scans with FIB, ranging from 10, 20 and 50, are made to achieve this dose. The GaN micromilling was investigated using $\mathrm{Ga}^{+}$ions with energy ranging from 30 to $70 \mathrm{keV}$, and $\mathrm{Au}^{+}$ions from 30 to $90 \mathrm{keV}$, respectively. The ion beam angle of incidence was varied between $0^{\circ}$ (normal incidence) and $30^{\circ}$ for $\mathrm{Ga}^{+}$, while $\mathrm{Au}^{+}$is incident to the normal surface of GaN.

The primary GaN material used for milling experiments was grown by hydride vapor phase epitaxy (HVPE) on sapphire substrates. The GaN film was relatively thick $(\sim 20 \mu \mathrm{m})$ which allowed for milling experiments to be performed without encountering any substrate effects. However, since in practice thinner GaN layers are grown on various substrates for fabricating devices, we have investigated the $\mathrm{FIB} \mathrm{Ga}^{+}$milling for the primary substrate material: c-face sapphire. We fabricated the waveguides and gratings on GaN (thickness $=2 \mu \mathrm{m}$ ), grown on sapphire by MOCVD.

Scanning electron microscopy (SEM) was used to observe the structures of laser cavity, mirror facets, and gratings on GaN samples. Another useful instrument to measure the depth profiles of milled pattern is the Atomic Force Microscope (AFM), which measures detailed information in the milling depth, side-wall angles and roughness of surface and bottom of the GaN pattern. AFM images and data were obtained by using Nanoscope II and Dimension 3100 systems from Digital Instruments.

\section{$\underline{\text { RESULTS }}$}

Fig. 1(a) shows an SEM microphotograph of a GaN pattern micromilled using $90 \mathrm{kV} \mathrm{Au}^{+}$ FIB with a dose of $1 \times 10^{18} \mathrm{~cm}^{-2}$, at normal incidence, and 10 FIB scans. The total area of the pattern FIB-micromilled into $\mathrm{GaN}$ is $200 \mu^{2}$, which requires approximately 30-35 min exposure for a $1 \times 10^{18} \mathrm{~cm}^{-2}$ dose. The milling depth of GaN experiments ranged between $\sim 0.6$ to $1 \mu \mathrm{m}$ for $\mathrm{Ga}^{+}$, and $\sim 2.3$ to $3 \mu \mathrm{m}$ for $\mathrm{Au}^{+}$. The "writing" strategy is that of serpentine scan, illustrated in 

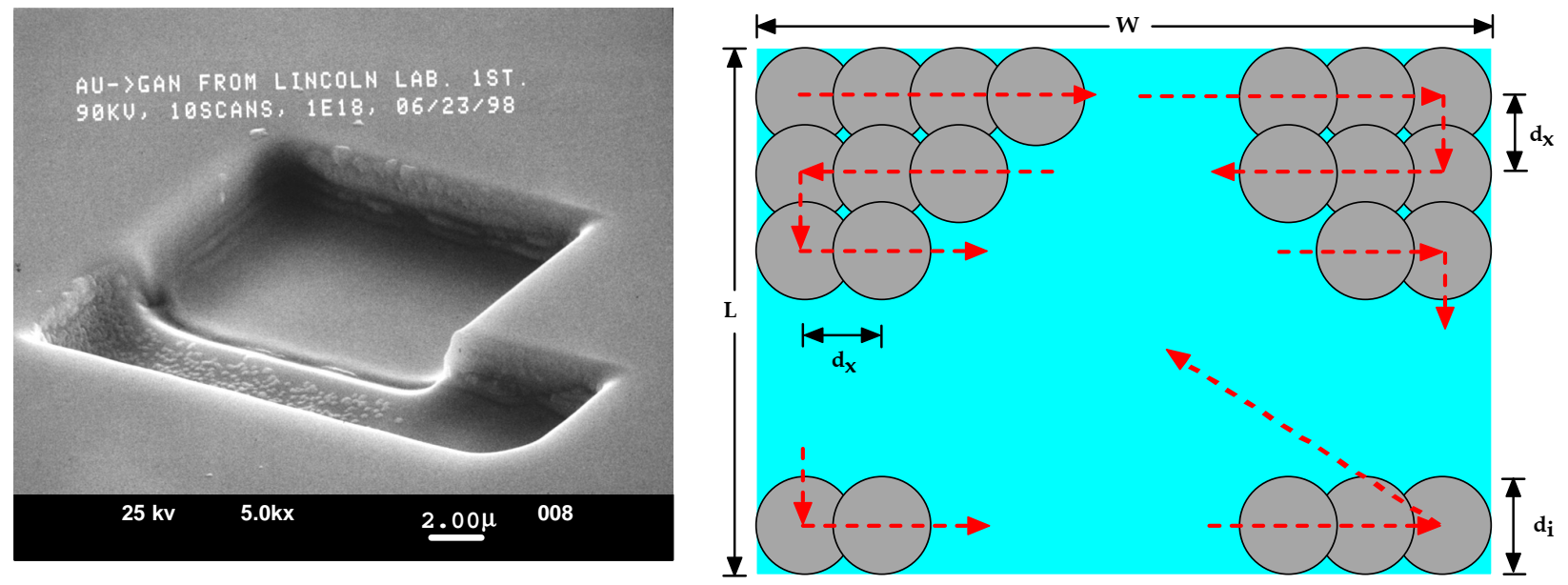

Fig. 1 The $\mathrm{Ga}^{+}$FIB micromachined pattern: (a) SEM photograph of GaN pattern micromachined using 90kV $\mathrm{Au}^{+} \mathrm{FIB}$ with a dose of $1 \times 10^{18} \mathrm{~cm}^{-2}$, at normal incidence, and $10 \mathrm{scans}$; (b) schematic diagram of serpentine scan.

Fig. 1(b), where the scan direction is reversed after each pass to minimize redeposition effects. A sharply defined milled region is obtained, with a uniform depth, a smooth milled surface, and with minimum redeposition of material. A deeper trench is observed where the two rectangles of the pattern have a slight overlap.

In Fig. 2, we compare the $\mathrm{Ga}^{+}$milling rate as a function of off-axis angle from the normal target surface at different energy from 30 to $70 \mathrm{keV}$ and $10 \mathrm{FIB}$ scans. We found that the milling rate increases with the $\mathrm{Ga}^{+}$incident angle in all cases. For the normal angle (i.e. $0^{\circ}$ ), increasing the ion energy results in an increasing the milling rate. For $15^{\circ}$ and $30^{\circ}$ the incident angles, the milling rate is weakly independent of ion energy. Fig. 3 shows the effect of changing the number of scans on the milling rate for various incident angles at a fixed ion energy of $50 \mathrm{keV}$. The milling rate is independent of FIB scans at $0^{\circ}$. For angles of $15^{\circ}$ and $30^{\circ}$, the milling rate becomes larger as the number of FIB scans increases. A similar effect was also observed at 30 and $70 \mathrm{keV}$.

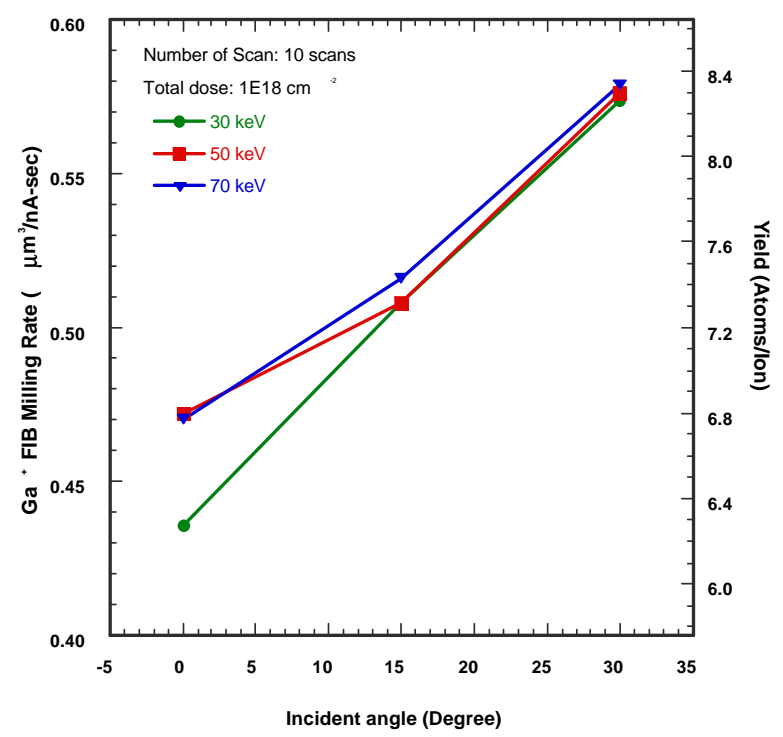

Fig. $2 \mathrm{Ga}^{+}$milling rate of $\mathrm{GaN}$ versus angle of incidence for different energies in 10 FIB scans. (total dose: $1 \times 10^{18} \mathrm{~cm}^{-2}$ )

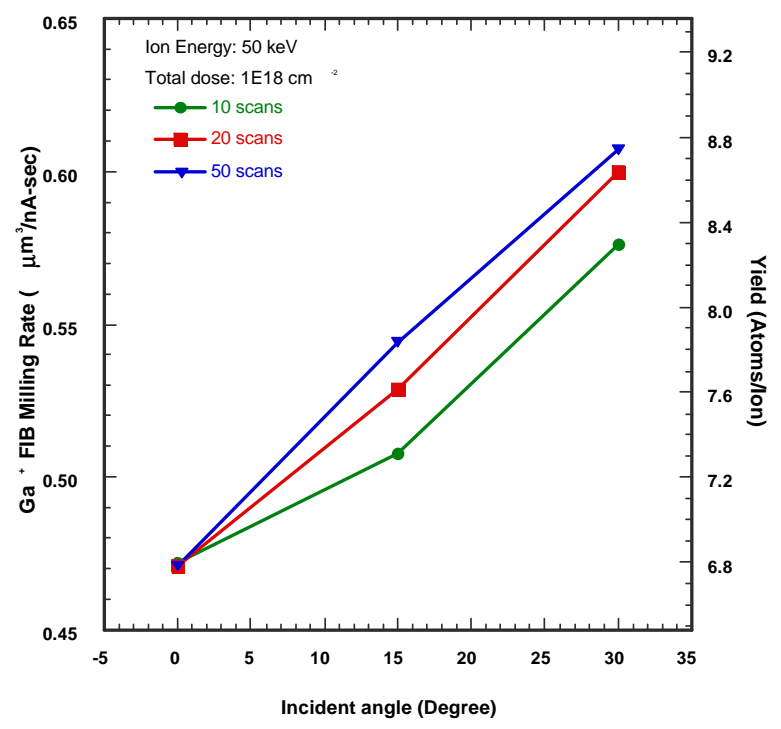

Fig. $3 \mathrm{Ga}^{+}$milling rate of $\mathrm{GaN}$ versus angle of incidence for different scan conditions at 50 $\mathrm{keV}$. (total dose: $1 \times 10^{18} \mathrm{~cm}^{-2}$ ) 


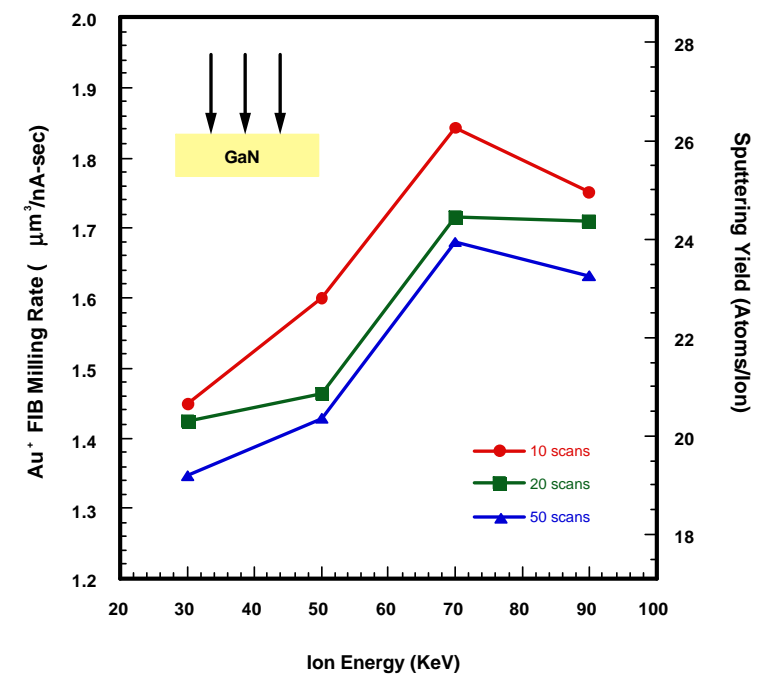

Fig. $4 \mathrm{Au}^{+}$milling rate of $\mathrm{GaN}$ versus ion energy for different scan conditions in the normal incidence. (total dose: $1 \times 10^{18} \mathrm{~cm}^{-2}$ )

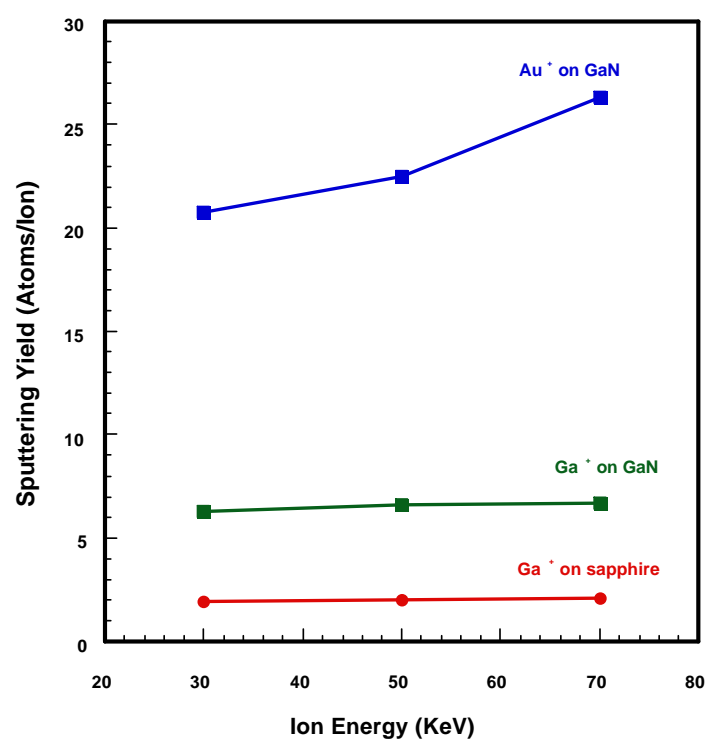

Fig. 5 Comparison of $\mathrm{Ga}^{+}$and $\mathrm{Au}^{+}$milling rate of $\mathrm{GaN}$ and sapphire as a function of ion energy. (total dose: $1 \times 10^{18} \mathrm{~cm}^{-2}$, normal incidence, 10 scans)

The milling rate of $\mathrm{Au}^{+}$ions for energies from 30 to $90 \mathrm{keV}$ and different repetitive scans under the condition of normal incident angle ( 0 degree) is observed in Fig. 4 . The milling rate increased with ion energy increasing from 30 to $70 \mathrm{keV}$, decreased at $90 \mathrm{keV}$. For $\mathrm{Au}^{+}$ion, the physical limitation of milling rate is at $70 \mathrm{keV}$. The reason is that for most of ion energy over 70 $\mathrm{keV}$, the ions will be implanted into the target instead of milling the target. The increase of scan speed (i.e. number of scans) reduces the milling rate for all energies. In Fig. 5, we compare the sputtering yield of $\mathrm{Au}^{+}$ions for $\mathrm{GaN}$ with $\mathrm{Ga}^{+}$ion for $\mathrm{GaN}$ and sapphire. As expected, the $\mathrm{Au}^{+}$ ion which is heavier than $\mathrm{Ga}^{+}$ion has a much higher sputtering yield. For example, the sputtering yield of $\mathrm{Au}^{+}$ion at conditions of $70 \mathrm{keV}$ and $10 \mathrm{FIB}$ scans is 26 atoms/ion, which is almost 4 times larger than $\mathrm{Ga}^{+}$ion under the same conditions. The drawbacks of $\mathrm{Au}^{+} \mathrm{FIB}$ milling add more redeposition on the side-wall and a wider beam diameter. From Fig. 1(a), we can see that there is some redeposition on the side-wall and bottom surface of the milled pattern. The $\mathrm{Ga}^{+}$sputtering yield of $\mathrm{GaN}$ is about 3 times larger than that of sapphire substrate. The fact that the GaN milling rate is always higher than sapphire is of assistance in terminating the milling process without removing excessive amounts of substrate material. We have observed that an increase in scan speed causes a decrease in sputtering yield of $\mathrm{Au}^{+}$ions at normal incidence, which is similar to the same result with $\mathrm{Ga}^{+}$ions shown in Fig. 3.

We have applied FIB milling to the fabrication of short cavity lasers which utilize air/semiconductor DBR grating mirrors. With this DBR structure, the mirrors formed by grating structures can offer better reflectivity than conventional cleaved mirrors. In Fig. 6, we show the SEM photographs for the DBR gratings and short cavity laser structures fabricated in GaN using $\mathrm{Ga}^{+}$ion FIB micromachining. The cavity length is $120 \mu \mathrm{m}$ and the cavity width is $18 \mu \mathrm{m}$, shown in Fig. 6(a). The DBR grating shown in Fig. 6(b) is a 5th order grating, with a grating period of $675 \mathrm{~nm}$ and spacing of $200 \mathrm{~nm}$. The SEM photographs indicate that FIB is a very precise technique for the fabrication of DBR gratings and other optoelectronic and photonic applications in GaN. 

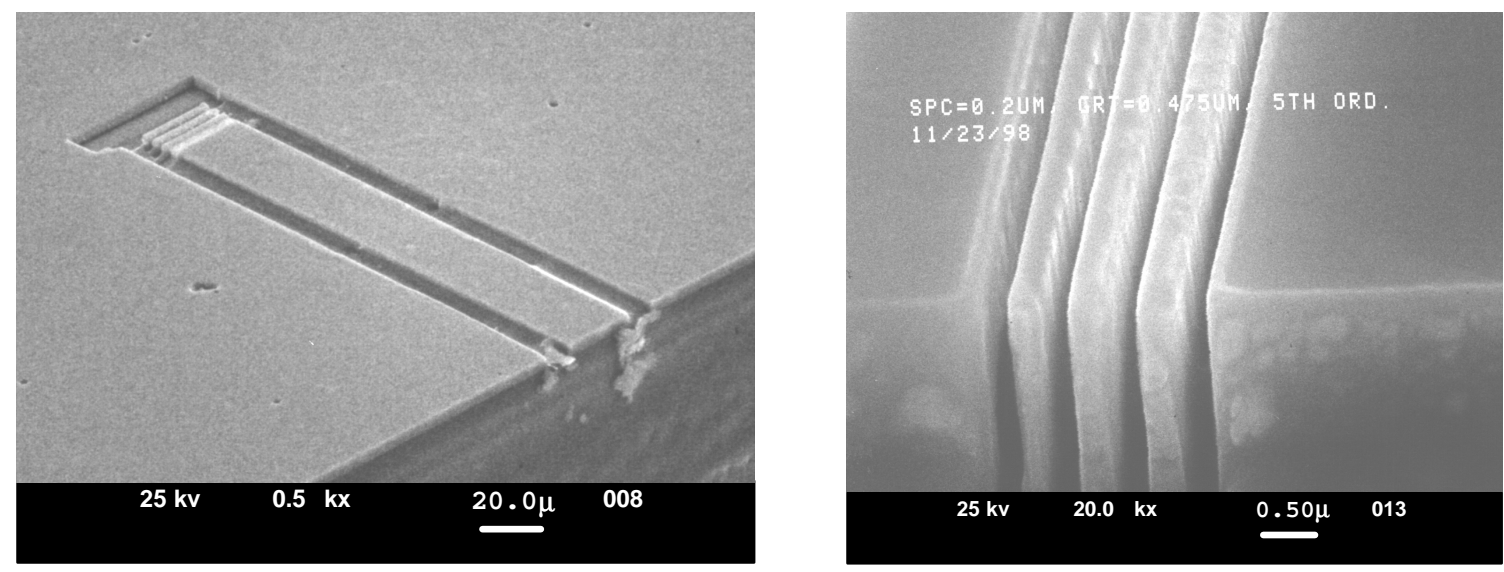

Fig. 6 The SEM photographs of the GaN laser with DBR gratings by FIB micromachining: (a) the overview of laser structure, cavity length is $120 \mu \mathrm{m}$; (b) the 5th order grating structure, the period is $675 \mathrm{~nm}$ and spacing is $200 \mathrm{~nm}$..

\section{CONCLUSION}

In conclusion, we have reported the $\mathrm{Ga}^{+}$and $\mathrm{Au}^{+} \mathrm{FIB}$ micromachining characteristics of GaN. We have compared the milling rate and sputtering yield of $\mathrm{GaN}$ by two potential ion species, $\mathrm{Ga}^{+}$and $\mathrm{Au}^{+}$, under various experimental conditions (ion energy, numbers of FIB scans, angle of incidence). The high FIB milling rate of GaN (significantly higher than that of common sapphire substrate) has positive implications for future fabrication of $\mathrm{GaN}$ devices. We also demonstrated that FIB micromachining has the capability to fabricate DBR gratings in order to obtain better reflectivity for laser diodes. We believe that FIB micromachining is a very promising technique for GaN laser fabrication. The authors gratefully acknowledge the HVPE GaN material provided by R. Molnar and the support of this work by MRL/NSA.

\section{REFERENCES}

${ }^{1}$ S. Nakamura, T. Mukai, and M. Senoh, Appl. Phys. Lett. 64, 1678 (1994).

2 J. Edgar, J. Mater. Res. 7, 235 (Jan. 1992).

${ }^{3}$ M. A. Khan, D. T. Olson, J. M. Van Hove, and J. N. Kuznia, Appl. Phys. Lett. 58, 1515 (1991).

${ }^{4}$ F. Binet, J. Y. Duboz, N. Laurent, C. Bonnat, P. Collot, F. Hanauer, O. Briot, and R. L. Aulombard, Appl. Phys. Lett. 72, 960 (1998).

${ }^{5}$ G. M. Smith, J. S. Hughes, M. L. Osowski, D. V. Forbes, and J. J. Coleman, Electron. Lett. 30, 30 (1990).

${ }^{6}$ D. Hofstetter, H. P. Zappe, J. E. Epler, and J. Söchtig, Electron. Lett. 30, 1858 (1990).

${ }^{7}$ T. Baba, M. Hamasaki, N. Watanabe, P. Kaewplung, A. Matsutani, T. Mukaihara, F. Koyama, and K. Iga, Jpn. J. Appl. Phys. Part 1, 35(2B), 1390 (1996).

${ }^{8}$ Y. Yuan, T. Brock, P. Bhattacharya, C. Caneau and R. Bhat, IEEE Photonics Technol. Lett., 9(7), 881 (1997).

${ }^{9}$ T. F. Krauss, O. Painter, A. Scherer, J. S. Roberts, and R. M. De La Rue, Opt. Eng., 37(4), 1143 (1998).

${ }^{10}$ L. R. Harriott and H. Temkin, Chapter 6 in Integrated Optoelectronics, edited by M. Dagenais, R. F. Leheny, and J. Crow, (Academic Press, New York, 1995).

${ }^{11}$ A. J. Steckl, P. Chen, H. E. Jackson, A. G. Choo, X. Cao, J. T. Boyd, and M. Kumar, J. Vac. Sci. Technol. B13, 2570 (1995). 
${ }^{12}$ T. Ito, H. Ishikawa, T. Egawa, T. Jimbo, M. Umeno, Jpn. J. Appl. Phys. Part 1, 36(12B), 7710 (1997).

${ }^{13}$ H. Katoh, T. Takeuchi, C. Anbe, R. Mizumotos, S. Yamaguchi, C. Weitzel, H. Amano, I. Akasaki, Y. Kaneko, N. Yamado, Jpn. J. Appl. Phys. Part 2, 36(4B), L444 (1998). 